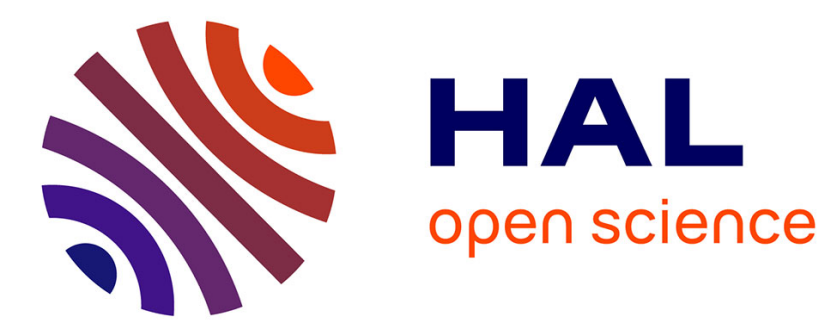

\title{
An optimized Schwarz algorithm for the compressible Euler equations
}

\author{
Victorita Dolean, Frédéric Nataf
}

\section{To cite this version:}

Victorita Dolean, Frédéric Nataf. An optimized Schwarz algorithm for the compressible Euler equations. Olof B. Widlund and David E. Keyes Edts. Domain decomposition methods in science and engineering XVI, Springer-Verlag, pp.173-180, 2007, LNCSE. hal-00413523

\section{HAL Id: hal-00413523 \\ https://hal.science/hal-00413523}

Submitted on 4 Sep 2009

HAL is a multi-disciplinary open access archive for the deposit and dissemination of scientific research documents, whether they are published or not. The documents may come from teaching and research institutions in France or abroad, or from public or private research centers.
L'archive ouverte pluridisciplinaire HAL, est destinée au dépôt et à la diffusion de documents scientifiques de niveau recherche, publiés ou non, émanant des établissements d'enseignement et de recherche français ou étrangers, des laboratoires publics ou privés. 


\title{
An optimized Schwarz algorithm for the compressible Euler equations
}

\author{
V. Dolean* and F. Nataf ${ }^{\dagger}$
}

5th May 2006

\begin{abstract}
In this work we design new interface transmission conditions for a domain decomposition Schwarz algorithm for the Euler equations in 2 dimensions. These new interface conditions are designed to improve the convergence properties of the Schwarz algorithm. These conditions depend on a few parameters and they generalize the classical ones. Numerical results illustrate the effectiveness of the new interface conditions.
\end{abstract}

\section{Introduction}

When solving the compressible Euler equations by an implicit scheme the nonlinear system is usually solved by Newton's method. At each step of this method we have to solve a linear system that is nonsymmetric and very ill conditioned. In a previous paper [DLN04] we formulated a Schwarz algorithm (interface iteration which relies on the successive solution of the local decomposed problems and the transmission of the result at the interface) involving transmission conditions that are derived naturally from a weak formulation of the underlying boundary value problem. We also studied the convergence of the proposed algorithm from a quantitative point of view in the two and three dimensional overlapping and non-overlapping cases by applying a Fourier analysis. For the sake of the analysis we limited ourselves to the cases of two and three subdomain decompositions and we provided analytical expressions of the convergence rate of the Schwarz algorithm applied to the linearized equations.

Various works and studies deal with Schwarz algorithms applied to scalar problems: classical and optimized transmission conditions as well as preconditionning aspects were treated for example in the case of the Poisson, advection-diffusion equations [JN00, JNR01], Helmholtz [GMN02], or other simple systems reducible to scalar problems, on conforming and non-conforming meshes. There are also such methods for linear systems such as time harmonic Maxwell equations [CDJP97][DJR92] [AG04] and linear elasticity. To our knowledge, little is known about multicomponent systems. When dealing with systems we can mention some classical works by Quarteroni and al. [Qua90] [QS96], Bjorhus [Bjø95] or Cai et al. [CFS98]. As far as the optimized interface conditions are concerned, we can mention [DLN02] based on the Smith factorization. The work most related to our study belongs to Clerc [Cle98] and it describes the principle of building very simple interface conditions for a general hyperbolic system which we will apply and extend to Euler system.

\footnotetext{
*Université d'Évry, Dept. Maths., 91000 Évry Cedex and CMAP, CNRS UMR7641,École Polytechnique, 91128 Palaiseau Cedex, France, dolean@cmapx.polytechnique.fr, http://www.cmap.polytechnique.fr/ dolean

${ }^{\dagger}$ CMAP, CNRS UMR7641, Ecole Polytechnique, 91128 Palaiseau, France, nataf@cmapx.polytechnique.fr, http://www.cmap.polytechnique.fr/ ${ }^{\text {nataf }}$
} 
In this work we formulate and analyze the convergence of the Schwarz algorithm with new interface conditions inspired by [Cle98], depending on two parameters whose value is determined by minimizing the norm of the convergence rate.

The paper is organized as follows. In the section 2 we first formulate the Schwarz algorithm for a general linear hyperbolic system of PDEs with general interface conditions built in order to have a wellposed problem. The convergence rate is computed in the Fourier space as a function of some parameters.

In the section 3 we present the discretization method as well as the discrete counterpart of the considered problem. We will further estimate the convergence rate at the discrete level. We will find the optimal parameters of the interface conditions at the discrete level.

In the section 4, we use the new optimal interface conditions in Euler computations which illustrate the improvement over the classical interface conditions (first described in [QS96]). An appendix containing the solution of the optimization problem in the non-overlapping case, where we can obtain some analytical results, concludes this work.

\section{A Schwarz algorithm with general interface conditions}

\subsection{A well-posed boundary value problem}

In this section we briefly review the main definitions and properties of hyperbolic systems of conservation laws that are of interest to our study. Then we introduce a Schwarz algorithm which is based on general transmission conditions at subdomain interfaces that take into account the hyperbolic nature of the problem. In addition, we recall some existing results concerning the convergence of the algorithm. We consider here a general nonlinear system of conservation laws which has the form:

$$
\frac{\partial W}{\partial t}+\sum_{i=1}^{d} \frac{\partial F_{i}(W)}{\partial x_{i}}=0, \quad W \in \mathbb{R}^{q}
$$

where $d$ denotes the space dimension and $q$ the dimension of the system. The flux functions $F_{i}$ are assumed differentiable with respect to the state vector $W=W(x, t)$. In the general case, the flux functions are non-linear functions of $W$. Under the hypothesis that the solution is regular, we can also write a nonconservative (or quasi-linear) equivalent form of equation (1) :

$$
\frac{\partial W}{\partial t}+\sum_{i=1}^{d} A_{i}(W) \frac{\partial W}{\partial x_{i}}=0
$$

where the $A_{i}$ are the Jacobian matrices of the flux vectors. Assume that we first proceed to an integration in time of (1) using a backward Euler implicit scheme involving a linearization of the flux functions and eventually we symmetrize it (we know that when the system admits an entropy it can be symmetrized by multiplying it by the Hessian matrix of this entropy). This operation results in the linearized system:

$$
\mathcal{L}(\delta W) \equiv \frac{\mathrm{Id}}{\Delta t} \delta W+\sum_{i=1}^{d} A_{i} \frac{\partial \delta W}{\partial x_{i}}=f,
$$

where $\delta W \equiv W^{n+1}-W^{n}$ and $W^{n+1}=W(x,(n+1) \Delta t)$, and $A_{i}$ is a shorthand for $A_{i}\left(W^{n}\right)$.

In the following we will define the boundary conditions that have to be imposed when solving the

problem on a domain $\Omega \subset \mathbb{R}^{d}$. We denote by $A_{\boldsymbol{n}}=\sum_{i=1}^{d} A_{i} n_{i}$, the linear combination of Jacobian matrices 
by the components of the outward normal vector at the boundary of the domain $\partial \Omega$. This matrix is real, symmetric and can be diagonalized

$$
A_{n}=T \Lambda_{n} T^{-1}, \Lambda_{n}=\operatorname{diag}\left(\lambda_{i}\right)
$$

It can also be split in negative and positive parts using this diagonalization

$$
\left\{\begin{array}{l}
A_{\boldsymbol{n}}=A_{\boldsymbol{n}}^{+}+A_{\boldsymbol{n}}^{-} \\
A_{\boldsymbol{n}}^{ \pm}=T \Lambda_{n}^{ \pm} T^{-1} \\
\Lambda_{n}^{+}=\operatorname{diag}\left(\max \left(\lambda_{i}, 0\right)\right), \Lambda_{n}^{-}=\operatorname{diag}\left(\min \left(\lambda_{i}, 0\right)\right)
\end{array}\right.
$$

This corresponds to a decomposition with local characteristic variables. A more general splitting in negative (positive) definite parts, $A_{\boldsymbol{n}}^{\text {neg }}$ and $A_{\boldsymbol{n}}^{\text {pos }}$, of $A_{\boldsymbol{n}}$ can be done such that these matrices satisfy the following properties:

$$
\begin{cases}A_{\boldsymbol{n}} & =A_{\boldsymbol{n}}^{\text {neg }}+A_{\boldsymbol{n}}^{\text {pos }} \\ \operatorname{rank}\left(A_{n}^{\text {neg,pos }}\right) & =\operatorname{rank}\left(A_{\boldsymbol{n}}^{ \pm}\right) \\ A_{-\boldsymbol{n}}^{\text {pos }} & =-A_{\boldsymbol{n}}^{\text {neg }}\end{cases}
$$

In the scalar case the only possible choice is $A_{\boldsymbol{n}}^{n e g}=A_{\boldsymbol{n}}^{-}$. Using the previous formalism we can define the following boundary condition:

$$
A_{\boldsymbol{n}}^{n e g} W=A_{\boldsymbol{n}}^{n e g} g, \text { on } \partial \Omega
$$

Remark 1 In the case of a classical decomposition into negative and positive part this boundary condition has the physical meaning of the incoming flux in domain $\Omega$. By extension of the properties found in this case we call the last equality of (4) conservation property because it insures that the "outflow" quantity (given by the positive part of the jacobian flux matrix with oposite direction of the normal) is retrieved out of the "inflow" quantity imposed by the boundary condition (given the negative part of the jacobian flux matrix).

Within this framework we have the following result concerning the boundary value problem associated to the system that can be found in [Cle98] :

Theorem 1 If $f \in L^{2}(\Omega)^{q}$ and $g$ satisfies $\left|\int_{\partial \Omega} A_{n}^{n e g} g \cdot g\right|<\infty$ and there exists a constant $C_{0}$ independent of $x$ such that the following inequality is respected in the sense of symmetric positive definite matrices:

$$
\left(\frac{I d}{\Delta t}-\sum \partial_{x_{i}} A_{i}\right) \geq C_{0} I d>0
$$

then there exists a unique, $W \in L^{2}(\Omega)^{q}$ with $\sum A_{i} \partial_{x_{i}} W \in L^{2}(\Omega)^{q}$ solution of the boundary value problem:

$$
\left\{\begin{aligned}
\mathcal{L}(W)=\frac{I d}{\Delta t} W+\sum_{i=1}^{d} A_{i} \partial_{x_{i}} W & =f \text { in } \Omega \\
A_{\boldsymbol{n}}^{n e g} W & =A_{\boldsymbol{n}}^{\text {neg } g} \text { on } \partial \Omega
\end{aligned}\right.
$$

The unique solution $W$ of (5) satisfies the estimate:

$$
C_{0}\|W\|_{L^{2}(\Omega)}^{2}+\int_{\partial \Omega} A_{\boldsymbol{n}}^{p o s} W \cdot W \leq-\int_{\partial \Omega} A_{\boldsymbol{n}}^{n e g} W \cdot W
$$

As the boundary value problem (5) is well-posed, the decomposition (4) enables the design of a domain decomposition method. 


\subsection{Schwarz algorithm with general interface conditions}

We consider a decomposition of the domain $\Omega$ into $N$ overlapping or non-overlapping subdomains $\bar{\Omega}=$ $\bigcup_{i=1}^{N} \bar{\Omega}_{i}$. We denote by $\boldsymbol{n}_{i j}$ the outward normal to the interface $\Gamma_{i j}$ bewteen $\Omega_{i}$ and a neighboring subdomain $\Omega_{j}$. Let $W_{i}^{(0)}$ denote the initial appoximation of the solution in subdomain $\Omega_{i}$. A general formulation of a Schwarz algorithm for computing $\left(W_{i}^{p+1}\right)_{1 \leq i \leq N}$ from $\left(W_{i}^{p}\right)_{1 \leq i \leq N}$ (where $p$ defines the iteration of the Schwarz algorithm) reads :

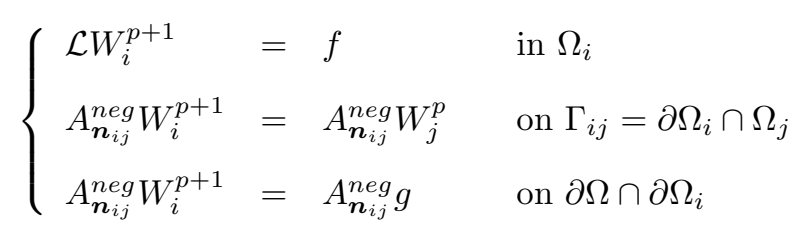

where $A_{\boldsymbol{n}_{i j}}^{n e g}$ and $A_{\boldsymbol{n}_{i j}}^{\text {pos }}$ satisfy (4). We have the following result concerning the convergence of the Schwarz algorithm in the non-overlapping case, due to([Cle98]):

Theorem 2 If we denote by $E_{i}^{p}=W_{i}^{p}-W_{i}$ the error vector associated to the restriction to the $i$-th subdomain of the global solution of the problem. Then, the Schwarz algorithm converges in the following sense :

$$
\left\{\begin{array}{l}
\lim _{p \rightarrow \infty}\left\|E_{i}^{p}\right\|_{L^{2}\left(\Omega_{i}\right)^{q}}=0 \\
\lim _{p \rightarrow \infty}\left\|\sum_{j=1}^{d} A_{j} \partial_{j} E_{i}^{p}\right\|_{L^{2}\left(\Omega_{i}\right)^{q}}=0
\end{array}\right.
$$

The convergence rate of the algorithm defined by (7) depends of the choice of the decomposition of $A_{\boldsymbol{n}_{i j}}$ into a negative and a positive part satisfying (4). In order to choose the decomposition (4) we need to relate this choice to the convergence rate of $(7)$.

\subsection{Convergence rate of the algorithm with general interface conditions}

We consider a two-subdomain non-overlapping or overlapping decomposition of the domain $\Omega=\mathbb{R}^{d}$, $\left.\Omega_{1}=\right]-\infty, \gamma\left[\times \mathbb{R}^{d-1}\right.$ and $\left.\Omega_{2}=\right] \beta, \infty\left[\times \mathbb{R}^{d-1}\right.$ with $\beta \leq \gamma$ and study the convergence of the Schwarz algorithm in the subsonic case. A Fourier analysis applied to the linearized equations allows us to derive the convergence rate of the " $\xi$ "-th Fourier component of the error. We will first briefly recall the technique of Fourier transform which was already described in detail in [DLN04]. The vector of Fourier variables is denoted by $\boldsymbol{\xi}=\left(\xi_{j}, j=2, \ldots, d\right)$. Let $\left(E_{i}^{p}\right)(x)=\left(W_{i}^{p}-W_{i}\right)(x)$ be the error vector in the $i$ th subdomain at the $p$ th iteration of the Schwarz algorithm and:

$$
\hat{E}\left(x_{1}, \xi_{2}, \ldots, \xi_{d}\right)=\mathcal{F} E\left(x_{1}, \xi_{2}, \ldots, \xi_{d}\right)=\int_{\mathbb{R}^{d-1}} e^{-i \xi_{2} x_{2}-\ldots-i \xi_{d} x_{d}} E\left(x_{1}, \ldots, x_{d}\right) d x_{2} \ldots d x_{d}
$$

be the Fourier symbol of the error vector. This transformation is useful only if the $A_{i}$ matrices are constant which is the case here because we have considered the linearized form of the Euler equations around a constant state $\bar{W}$. The Schwarz algorithm in the Fourier space $\left(\xi \in \mathbb{R}^{d-1}\right)$ can be written as follows:

$$
\left\{\begin{array} { l } 
{ \frac { d } { d x _ { 1 } } \hat { E } _ { 1 } ^ { p + 1 } = - M ( \boldsymbol { \xi } ) \hat { E } _ { 1 } ^ { p + 1 } , x < \gamma } \\
{ \mathcal { A } ^ { \text { neg } } ( \hat { E } _ { 1 } ^ { p + 1 } ) = \mathcal { A } ^ { \text { neg } } ( \hat { E } _ { 2 } ^ { p } ) , \text { on } x = \gamma }
\end{array} \left\{\begin{array}{l}
\frac{d}{d x_{1}} \hat{E}_{2}^{p+1}=-M(\boldsymbol{\xi}) \hat{E}_{2}^{p+1}, x>\beta \\
\mathcal{A}^{\text {pos }}\left(\hat{E}_{2}^{p+1}\right)=\mathcal{A}^{\text {pos }}\left(\hat{E}_{1}^{p}\right), \text { on } x=\beta
\end{array}\right.\right.
$$


where we denoted by $\mathcal{A}^{\text {neg }}=A_{\boldsymbol{n}}^{\text {neg }}, \mathcal{A}^{\text {pos }}=A_{\boldsymbol{n}}^{\text {pos }}$ with $\boldsymbol{n}=(1, \mathbf{0})$ the outward normal to the domain $\Omega_{1}$ and:

$$
M(\boldsymbol{\xi})=A_{1}^{-1}\left(\frac{1}{\Delta t} \operatorname{Id}+\sum_{i=2}^{d} A_{i} \xi_{i-1}\right)
$$

We thus obtain local problems that for a given $\xi$ are simple ODEs whose solutions can be expressed as linear combinations of the eigenvectors of $M(\boldsymbol{\xi})$ :

$$
\hat{E}_{i}^{p}\left(x_{1}, \boldsymbol{\xi}\right)=\sum_{j=1}^{q} \alpha_{j}^{i, p}(\boldsymbol{\xi}) e^{-\lambda_{j}(\boldsymbol{\xi}) x_{1}} V_{j}(\boldsymbol{\xi})
$$

where $\lambda_{j}(\boldsymbol{\xi})$ are the eigenvalues of $M(\boldsymbol{\xi})$. Here we have assumed that the eigenvectors $V_{j}(\boldsymbol{\xi})$ of $M(\boldsymbol{\xi})$ are linearly independent. Furthermore, we require that these solutions are bounded at infinity $(-\infty$ and $+\infty$ respectively). We deduce that in the decomposition of $\hat{E}_{1}\left(x_{1}, \boldsymbol{\xi}\right)$ (respectively $\hat{E}_{2}\left(x_{1}, \boldsymbol{\xi}\right)$ ) we must keep only the eigenvectors corresponding to the negative (respectively the positive) real parts of the eigenvalues. Taking into account these considerations we replace the expressions of the local solutions (10) into the interface conditions (8) to obtain the interface iterations on the $\alpha$ coefficients:

$$
\left\{\begin{aligned}
\left(\alpha_{j}^{1, p+1}\right)_{j, \Re\left(\lambda_{j}\right)<0}(\boldsymbol{\xi}) & =\mathcal{T}_{1}\left[\left(\alpha_{j}^{2, p}\right)_{j, \Re\left(\lambda_{j}\right)>0}(\boldsymbol{\xi})\right] \\
\left(\alpha_{j}^{2, p+1}\right)_{j, \Re\left(\lambda_{j}\right)>0}(\boldsymbol{\xi}) & =\mathcal{T}_{2}\left[\left(\alpha_{j}^{1, p}\right)_{j, \Re\left(\lambda_{j}\right)<0}(\boldsymbol{\xi})\right]
\end{aligned}\right.
$$

Then, the convergence rate of the $\boldsymbol{\xi}$-th component of the error vector of the Schwarz algorithm can be computed as the spectral radius of one of the iteration matrices $\mathcal{T}_{1} \mathcal{T}_{2}(\boldsymbol{\xi})$ or $\mathcal{T}_{2} \mathcal{T}_{1}(\boldsymbol{\xi})$ :

$$
\rho_{2}^{2} \equiv \rho_{\text {Schwarz } 2}^{2}=\rho\left(\mathcal{T}_{1} \mathcal{T}_{2}\right)=\rho\left(\mathcal{T}_{2} \mathcal{T}_{1}\right)
$$

\subsection{The 2D Euler equations}

After having defined in a general frame the well-posedness of the boundary value problem associated to a general equation and the convergence of the Schwarz algorithm applied to this class of problems, we will concentrate on the conservative Euler equations in two dimensions:

$$
\frac{\partial W}{\partial t}+\nabla \cdot \boldsymbol{F}(W)=0 \quad, \quad W=(\rho, \rho \boldsymbol{V}, E)^{T}, \quad \nabla=\left(\frac{\partial}{\partial x}, \frac{\partial}{\partial y}\right)^{T} .
$$

In the above expressions, $\rho$ is the density, $\boldsymbol{V}=(u, v)^{T}$ is the velocity vector, $E$ is the total energy per unit of volume and $p$ is the pressure. In equation $(11), W=W(\mathbf{x}, \mathbf{t})$ is the vector of conservative variables, $\mathbf{x}$ and $t$ respectively denote the space and time variables and $\boldsymbol{F}(W)=\left(F_{1}(W), F_{2}(W)\right)^{T}$ is the conservative flux vector whose components are given by

$$
F_{1}(W)=\left(\begin{array}{c}
\rho u \\
\rho u^{2}+p \\
\rho u v \\
u(E+p)
\end{array}\right), \quad F_{2}(W)=\left(\begin{array}{c}
\rho v \\
\rho u v \\
\rho v^{2}+p \\
v(E+p)
\end{array}\right) .
$$

The pressure is deduced from the other variables using the state equation for a perfect gas $p=$ $\left(\gamma_{s}-1\right)\left(E-\frac{1}{2} \rho\|\boldsymbol{V}\|^{2}\right)$ where $\gamma_{s}$ is the ratio of the specific heats $\left(\gamma_{s}=1.4\right.$ for the air $)$. 


\subsection{A new type of interface conditions}

We will apply now the method described previously to the computation of the convergence rate of the Schwarz algorithm applied to the two-dimensional subsonic Euler equations. In the supersonic case there is only one decomposition satisfying (4), that is: $\mathcal{A}^{\text {pos }}=A_{n}$ and $\mathcal{A}^{\text {neg }}=0$ and the convergence follows in 2 steps. Therefore the only case of interest is the subsonic one.

The starting point of our analysis is given by the linearized form of the Euler equations (11) which are of the form (3) where we replace $\delta W$ by $W$ and to which we applied a change of variable $\tilde{W}=T^{-1} W$ based on the eigenvector factorization of $A_{1}=T \tilde{A}_{1} T^{-1}$. In the following we will abandon the ${ }^{\sim}$ symbol:

$$
\frac{W}{c \Delta t}+A_{1} \partial_{x} W+A_{2} \partial_{y} W=0
$$

characterized by the following jacobian matrices:

$$
A_{2}=\left(\begin{array}{cccc}
M_{t} & 0 & \frac{1}{\sqrt{2}} & 0 \\
0 & M_{t} & \frac{1}{\sqrt{2}} & 0 \\
\frac{1}{\sqrt{2}} & \frac{1}{\sqrt{2}} & M_{t} & 0 \\
0 & 0 & 0 & M_{t}
\end{array}\right)
$$

where $M_{n}=\frac{u}{c}, M_{t}=\frac{v}{c}$ denote respectively the normal and the tangential Mach number. Before estimating the convergence rate we will derive the general transmission conditions at the interface by splitting the matrix $A_{1}$ into a positive and negative part.

We have the following general result concerning this decomposition:

Lemma 1 Let $\lambda_{1}=M_{n}-1, \lambda_{2}=M_{n}+1, \lambda_{3}=\lambda_{4}=M_{n}$. Suppose we deal with a subsonic flow: $0<u<c$ so that $\lambda_{1}<0, \lambda_{2,3,4}>0$. Any decomposition of $A_{1}=A_{\boldsymbol{n}}, \boldsymbol{n}=(1,0)$ which satisfies (4) has to be of the form:

$$
\begin{aligned}
& \mathcal{A}^{\text {neg }}=\frac{1}{a_{1}} \mathbf{u} \cdot \mathbf{u}^{t}, \mathbf{u}=\left(a_{1}, a_{2}, a_{3}, a_{4}\right)^{t} \\
& \mathcal{A}^{\text {pos }}=A_{\boldsymbol{n}}-\mathcal{A}^{\text {neg }} .
\end{aligned}
$$

where $\left(a_{1}, a_{2}, a_{3}, a_{4}\right) \in \mathbb{R}^{4}$ satisfies $a_{1} \leq \lambda_{1}<0$ and $\frac{a_{1}}{\lambda_{1}}+\frac{a_{2}^{2}}{a_{1} \lambda_{2}}+\frac{a_{3}^{2}}{a_{1} \lambda_{3}}+\frac{a_{4}^{2}}{a_{1} \lambda_{4}}=1$.

Proof The fact that each 1-rank symmetric matrix is of the form $\pm \mathbf{v} \cdot \mathbf{v}^{t}$ is straightforward. In order to have a negative matrix we need to take: $\mathcal{A}^{\text {neg }}=-\mathbf{v} \cdot \mathbf{v}^{t}$ with $\mathbf{v}=\left(c_{1}, c_{2}, c_{3}, c_{4}\right)^{t}$. Indeed, for each vector $\mathbf{x}=\left(x_{1}, x_{2}, x_{3}, x_{4}\right)^{t}$ we have:

$$
\mathbf{x}^{t} \mathcal{A}^{\text {neg }} \mathbf{x}=-\left(\mathbf{x}^{t} \cdot \mathbf{v}\right)^{2} \leq 0
$$

A necessary condition for $\mathcal{A}^{\text {pos }}$ to be of rank 3 is $\operatorname{det}\left(A_{\boldsymbol{n}}-\mathcal{A}^{\text {neg }}\right)=0$, that is:

$$
0=\frac{c_{1}^{2}}{\lambda_{1}}+\frac{c_{2}^{2}}{\lambda_{2}}+\frac{c_{3}^{2}}{\lambda_{3}}+\frac{c_{4}^{2}}{\lambda_{3}}+1 \geq 1+\frac{c_{1}^{2}}{\lambda_{1}}
$$

which implies that $c_{1} \neq 0$. Thus, without loss of generality and in order to simplify the writing of the interface conditions, in the sequel we will take $\mathcal{A}^{\text {neg }}=\frac{1}{a_{1}} \mathbf{u} \cdot \mathbf{u}^{t}$ with $\mathbf{u}=\left(a_{1}, a_{2}, a_{3}, a_{4}\right)^{t}$. On the other hand,

$$
\mathcal{A}^{\text {pos }}=A_{n}-\mathcal{A}^{\text {neg }}
$$


is of a maximum $\operatorname{rank} 3 \operatorname{iff} \operatorname{det}\left(\mathcal{A}^{\text {pos }}\right)=0$, that is:

$$
\frac{a_{1}}{\lambda_{1}}+\frac{a_{2}^{2}}{a_{1} \lambda_{2}}+\frac{a_{3}^{2}}{a_{1} \lambda_{3}}+\frac{a_{4}^{2}}{a_{1} \lambda_{3}}=1 .
$$

In the same time from $A_{\boldsymbol{n}}=\mathcal{A}^{\text {pos }}+\mathcal{A}^{\text {neg }}$ and the fact that $\operatorname{rank}\left(\mathcal{A}^{\text {neg }}\right)=1$ and $\operatorname{rank}\left(A_{\boldsymbol{n}}\right)=4$ we infer that $\operatorname{rank}\left(\mathcal{A}^{\text {pos }}\right) \geq 3$, therefore $\operatorname{rank}\left(\mathcal{A}^{\text {neg }}\right)=3$. Relation (15) also implies that

$$
\lambda_{1}-a_{1}=\frac{\lambda_{1}}{a_{1}}\left(\frac{a_{2}^{2}}{\lambda_{2}}+\frac{a_{3}^{2}}{\lambda_{3}}+\frac{a_{4}^{2}}{\lambda_{3}}\right) \geq 0 .
$$

Under these hypothesis we can show that $\mathcal{A}^{\text {pos }}$ is positive. First of all we can see that if $a_{1}=\lambda_{1}$ this result is obvious as we are in the case of the classical transmission conditions. Suppose now that $\left.a_{1} \in\right]-\infty, \lambda_{1}[$. Then, by using the above relation and the Cauchy-Schwarz inequality applied to the vectors $\left(\frac{a_{2}}{\sqrt{\lambda_{2}}}, \frac{a_{3}}{\sqrt{\lambda_{3}}}, \frac{a_{4}}{\sqrt{\lambda_{4}}}\right)$ and $\left(x_{2} \sqrt{\lambda_{2}}, x_{3} \sqrt{\lambda_{3}}, x_{4} \sqrt{\lambda_{4}}\right)$ :

$$
\frac{a_{1}}{\lambda_{1}}\left(\lambda_{1}-a_{1}\right)\left(\lambda_{2} x_{2}^{2}+\lambda_{3} x_{3}^{2}+\lambda_{3} x_{4}^{2}\right)=\left(\frac{a_{2}^{2}}{\lambda_{2}}+\frac{a_{3}^{2}}{\lambda_{3}}+\frac{a_{4}^{2}}{\lambda_{3}}\right)\left(\lambda_{2} x_{2}^{2}+\lambda_{3} x_{3}^{2}+\lambda_{3} x_{4}^{2}\right) \geq\left(a_{2} x_{2}+a_{3} x_{3}+a_{4} x_{4}\right)^{2} .
$$

From the previous inequality we have a minoration of $\lambda_{2} x_{2}^{2}+\lambda_{3} x_{3}^{2}+\lambda_{3} x_{4}^{2}$ by a term in $y=a_{2} x_{2}+a_{3} x_{3}+a_{4} x_{4}$ and then by using it we get the desired result:

$$
\begin{aligned}
\mathbf{x}^{t} \mathcal{A}^{\text {pos }} \mathbf{x} & =\left(\lambda_{1}-a_{1}\right) x_{1}^{2}-2 x_{1} y-\frac{1}{a_{1}} y^{2}+\lambda_{2} x_{2}^{2}+\lambda_{3} x_{3}^{2}+\lambda_{3} x_{4}^{2} \\
& \geq\left(\lambda_{1}-a_{1}\right) x_{1}^{2}-2 x_{1} y+\frac{y^{2}}{\lambda_{1}-a_{1}}=\left(\sqrt{\lambda_{1}-a_{1}} x_{1}-\frac{y}{\sqrt{\lambda_{1}-a_{1}}}\right)^{2} \geq 0
\end{aligned}
$$

We will proceed now to the estimation of the convergence rate using some results from [DLN04]. The matrix $M$ corresponding to (9) is written as:

$$
M(\xi)=\left(\begin{array}{cccc}
\frac{a}{M_{n}-1} & 0 & \frac{i \xi}{\sqrt{2}\left(M_{n}-1\right)} & 0 \\
0 & \frac{a}{1+M n} & \frac{i \xi}{\sqrt{2}(1+M n)} & 0 \\
\frac{i \xi}{\sqrt{2} M_{n}} & \frac{i \xi}{\sqrt{2} M_{n}} & \frac{a}{M_{n}} & 0 \\
0 & 0 & 0 & \frac{a}{u}
\end{array}\right)
$$

with $\beta=\frac{1}{c \Delta t}, a=\beta+i \xi M_{t}$. We obtain the following expressions for the eigenvalues and the corresponding eigenvectors of the matrix $M(\xi)$ :

$$
\begin{gathered}
\lambda_{1}(\xi)=\frac{-a M_{n}-R(\xi)}{1-M_{n}^{2}}, V_{1}(\xi)=\left[-\frac{(R(\xi)+a)\left(1+M_{n}\right)}{\sqrt{2}}, \frac{(R(\xi)-a)\left(1-M_{n}\right)}{\sqrt{2}}, i \xi\left(1-M_{n}^{2}\right), 0\right]^{T} \\
\lambda_{2}(\xi)=\frac{-a M_{n}+R(\xi)}{1-M_{n}^{2}}, V_{2}(\xi)=\left[\frac{(R(\xi)-a)\left(1+M_{n}\right)}{\sqrt{2}},-\frac{(R(\xi)+a)\left(1-M_{n}\right)}{\sqrt{2}}, i \xi\left(1-M_{n}^{2}\right), 0\right]^{T} \\
\lambda_{3,4}(\xi)=\frac{a}{M_{n}}, V_{3}(\xi)=\left[-\frac{i \xi M_{n}}{\sqrt{2}}, \frac{i \xi M_{n}}{\sqrt{2}}, a, 0\right]^{T}, V_{4}(\xi)=[0,0,0,1]^{T}
\end{gathered}
$$


where $R(\xi)=\sqrt{a^{2}+\xi^{2}\left(1-M_{n}^{2}\right)}$. We recall that we made the assumption that the flow is subsonic i.e. $M<1$; this also means that $M_{n}<1$ and $M_{t}<1$ since $M^{2}=M_{n}^{2}+M_{t}^{2}$. Finally we also assume that the flow is such that $u>0$, in other words we have that $0<M_{n}<1$. Under the assumption $0<u<c$ we have that $\Re\left(\lambda_{1}(\xi)\right)<0$ and $\Re\left(\lambda_{2,3,4}(\xi)\right)>0$.

Following the technique described in section 2.3 we estimate the convergence rate in the non-overlapping case and we use the non-dimensional wave-number $\bar{\xi}=c \Delta t \xi$. If we drop the bar symbol, we get for the general interface conditions the following:

$$
\begin{cases}\rho_{2, n o v r}^{2}(\xi) & =\left|1-\frac{4 M_{n}\left(1-M_{n}\right)\left(1+M_{n}\right) R(\xi) a_{1}^{2}\left(a+M_{n} R(\xi)\right)}{D_{1} D_{2}}\right| \\ D_{1} & =R(\xi)\left[a_{1}\left(1+M_{n}\right)-a_{2}\left(1-M_{n}\right)\right]+a\left[a_{1}\left(1+M_{n}\right)+a_{2}\left(1-M_{n}\right)\right]-i \sqrt{2} a_{3} \xi\left(1-M_{n}^{2}\right) \\ D_{2} & =M_{n} a_{1}\left[R(\xi)\left[a_{1}\left(1+M_{n}\right)-a_{2}\left(1-M_{n}\right)\right]+a\left[a_{1}\left(1+M_{n}\right)+a_{2}\left(1-M_{n}\right)\right]\right] \\ & +a_{3}\left(1-M_{n}^{2}\right)\left[a_{3}(R+a)-i M_{n} a_{1} \xi \sqrt{2}\right]\end{cases}
$$

Remark 2 The expression (17) gives the convergence rate in the classical case for $a_{1}=-\left(1-M_{n}\right)=$ $\lambda_{1}(0)$ and $a_{2}=a_{3}=a_{4}=0$, which corresponds to the classical transmission conditions. Moreover, theorem 2 proves that this quantity is always strictly less than 1 as the algorithm is convergent.

In order to simplify our optimization problem we will take $a_{3}=0$, we can thus reduce the number of parameters to $2, a_{1}$ and $a_{2}$, as we can see from (15) that $a_{4}$ can be expressed as a function of $a_{1}, a_{2}$ and $a_{3}$. We can also see that the convergence rate is a real quantity when the flow is normal to the interface $M_{t}=0$. In the same time for the purpose of optimization only we introduce the parameters: $b_{1}=-a_{1} /\left(1-M_{n}\right)$ and $b_{2}=a_{2} /\left(1+M_{n}\right)$ which provide a simpler form of the convergence rate:

$$
\rho_{2, \text { novr }}^{2}(\xi)=\left|1-\frac{4 b_{1}\left(a+M_{n} R(\xi)\right) R(\xi)}{\left(R(\xi)\left(b_{1}+b_{2}\right)+a\left(b_{1}-b_{2}\right)\right)^{2}(M n+1)}\right|
$$

From (15) we get the intervals in which the new parameters lie:

$$
\left\{\begin{array}{l}
\left.b_{1} \in \mathcal{I}_{1}=\right] 1, \infty\left[, b_{2} \in \mathcal{I}_{2}\left(b_{1}\right)\right. \\
b_{2}^{2} \leq \frac{1-M_{n}}{1+M_{n}} b_{1}\left(b_{1}-1\right) \Rightarrow \mathcal{I}_{2}\left(b_{1}\right)=\left[-\sqrt{\frac{1-M_{n}}{1+M_{n}} b_{1}\left(b_{1}-1\right)}, \sqrt{\frac{1-M_{n}}{1+M_{n}} b_{1}\left(b_{1}-1\right)}\right]
\end{array}\right.
$$

Before proceeding to the analysis of the general case we recall some results found in the classical case obtained in [DLN04]. The asymptotic convergence rate in the non-overlapping case:

$$
\lim _{k \rightarrow+\infty} \rho_{2, n o v r}(k)=\sqrt{\left(\frac{1-3 M_{n}}{1+M_{n}}\right)^{2}+\frac{8 M_{n} M_{t}^{2}}{\left(1+M_{n}\right)^{3}}}<1
$$

is always strictly inferior to 1 . Moreover, in the particular case $M_{n}^{\star}=1 / 3$ and $M_{t}=0$, this limit becomes null. The inequality (20) has a numerical meaning. For a given discretization, let $\xi_{\text {max }}$ denote the largest frequency supported by the numerical grid. This largest frequency is of the order $\pi / h$ with $h$ a typical mesh size. The convergence rate in a numerical computation made on this grid can be estimated by $\rho_{2}^{h}=\max _{|\xi|<\xi_{\max }} \rho_{2}(\xi)$. From $(20)$, we have that $\rho_{2}^{h} \leq \max _{|\xi|<\xi_{\max }} \rho_{2}(\xi)<1$. This means that for finer and finer grids, the number of iterations may increase slightly but should not go to infinity. Thus the optimization problem with respect to the parameters $b_{1}$ and $b_{2}$, makes sense:

$$
\min _{\left(b_{1}, b_{2}\right) \in \mathcal{I}_{1} \times \mathcal{I}_{2}\left(b_{1}\right)} \max _{\xi \geq 0} \rho(\xi)
$$


The solution of this problem is quite a tedious task even in the non-overlapping case, where we can obtain analytical expression of the parameters only for some values of the Mach number (see the appendix for details). In the same time, we have to analyze the convergence of the overlapping algorithm. Indeed, standard discretizations of the interface conditions correspond to overlapping decompositions with an overlap of size $\delta=h, h$ being the mesh size, as seen in [CFS98] and [DLN04]. By applying the procedure described in section 2.3 to the overlapping case we have the following expression of the convergence rate:

$$
\left\{\begin{aligned}
\rho_{2, \text { ovr }}^{2} & =\left|A e^{-\left(\lambda_{2}(k)-\lambda_{1}(k)\right) \bar{\delta}}+(B+C) e^{-\left(\lambda_{3}(k)-\lambda_{1}(k)\right) \bar{\delta}}\right| \\
A & =\frac{a+M_{n} R(\xi)}{a-M_{n} R(\xi)} \cdot\left(\frac{b_{1}(R(\xi)-a)+b_{2}(R(\xi)+a)}{b_{1}(R(\xi)+a)+b_{2}(R(\xi)-a)}\right)^{2} \\
B & =-\frac{2 M_{n}\left(b_{1}\left(1-M_{n}\right)+b_{2}\left(1+M_{n}\right)\right) R(\xi)(R(\xi)-a)(R(\xi)+a)}{\left(1-M_{n}^{2}\right)\left(a-M_{n} R(\xi)\right)\left(b_{1}(R(\xi)+a)+b_{2}(R(\xi)-a)\right)^{2}} \\
C & =\frac{4\left(\left(1-M_{n}\right)\left(b_{1}^{2}-b_{1}\right)-b_{2}^{2}(M n+1)\right)\left(a+M_{n} R(\xi)\right)}{\left(1-M_{n}^{2}\right)\left(b_{1}(R(\xi)+a)+b_{2}(R(\xi)-a)\right)^{2}}
\end{aligned}\right.
$$

where $\bar{\delta}=\frac{\delta}{c \Delta t}$ denotes the non-dimensional overlap between subdomains.

Analytic optimization with respect to $b_{1}$ and $b_{2}$ seems out of reach. We will have to use numerical procedures of optimization. In order to get closer to the numerical simulations we will estimate the convergence rate for the discretized equations with general transmission conditions, both in the non-overlapping and the overlapping case and then optimize numerically this quantity in order to get the best parameters for the convergence.

\section{Optimized interface conditions}

In this section we study the convergence of the Schwarz algorithm with general interface conditions applied to the discrete Euler equations. First, we consider a well-posed boundary value problem defined on a half plane as described in [DLN04]. This BVP is discretized using a finite volume scheme where the flux at the interface of the finite volume cells is computed using a Roe [Roe81] type solver. Afterwards, we formulate a Schwarz algorithm whose convergence rate is estimated in a discrete context.

\subsection{Discretization by a finite volume method}

We consider first the following BVP defined on the domain $\left.\Omega_{1}=\right]-\infty, \gamma[\times \mathbb{R}$

$$
\left\{\begin{array}{l}
\frac{W}{c \Delta t}+A_{1} \frac{\partial W}{\partial x}+A_{2} \frac{\partial W}{\partial y}=f, \quad \text { for } \quad x<\gamma \\
\mathcal{A}^{\text {neg }} W=g \text { for } x=\gamma
\end{array}\right.
$$

In order to discretize the BVP (23) we consider a regular quadrilateral grid where a vertex $v_{i j}$ is characterized by

$$
v_{i j}=\left(\left(i-\frac{1}{2}\right) \Delta x,\left(j-\frac{1}{2}\right) \Delta y\right) \quad \text { for } \quad i \leq 0 \quad \text { and } \quad j \in \mathbb{Z} .
$$

We associate to each vertex a finite volume cell, $C_{i j}=[(i-1) \Delta x, i \Delta x] \times[(j-1) \Delta x, j \Delta x]$ which is a rectangle having as a center the vertex $v_{i j}$. A first order vertex centered finite volume formulation for the discretization of (23) simply is written (see for example [Cle98])

$$
\frac{W_{i, j}}{c \Delta t}+\frac{1}{\left|C_{i j}\right|} \sum_{e \in \partial C_{i j}}|e| \Phi^{e}=f
$$


where $\left|C_{i j}\right|$ denotes the area of the cell $C_{i j}$, $|e|$ the length of the edge $e$ and $W_{i, j}$ the average value of the unknown on the cell $C_{i j}$

$$
W_{i, j}=\frac{1}{\left|C_{i j}\right|} \int_{C_{i j}} W(x, y) d x d y .
$$

Here, the elementary flux $\Phi_{i j}^{e}$ across edge $|e|$ is computed by a Roe type scheme

$$
\Phi^{e}=A_{n}^{+} W_{i, j}+A_{n}^{-} W_{k, l},
$$

where $\boldsymbol{n}=\left(n_{x}, n_{y}\right)$ is the outward normal to the the edge $e, A_{\boldsymbol{n}}=n_{x} A_{1}+n_{y} A_{2}$ and $C_{k l}$ is the neighboring cell of $C_{i j}$ sharing the edge $e$ with it. In the present case, we easily see that $C_{k l}$ is such that $(k, l) \in\{(i-1, j),(i+1, j),(i, j-1),(i, j+1)\}$ and the four edges of a cell have the following lengths and outward normal vectors

$$
\begin{aligned}
& \left|e_{1}\right|=\Delta x, \boldsymbol{n}_{1}=(0,1),\left|e_{2}\right|=\Delta y, \boldsymbol{n}_{2}=(1,0) \\
& \left|e_{3}\right|=\Delta x, \boldsymbol{n}_{3}=(0,-1),\left|e_{4}\right|=\Delta y, \boldsymbol{n}_{4}=(-1,0)
\end{aligned}
$$

which allows us to rewrite $(24)$ as

$$
\frac{W_{i j}}{c \Delta t}+\frac{\left|A_{1}\right| W_{i, j}+A_{1}^{-} W_{i+1, j}-A_{1}^{+} W_{i-1, j}}{\Delta x}+\frac{\left|A_{2}\right| W_{i, j}+A_{2}^{-} W_{i, j+1}-A_{2}^{+} W_{i, j-1}}{\Delta y}=f, i \leq l_{2} . .
$$

where $\gamma=l_{2} \Delta x$. We will further denote $\bar{\Delta} x=\frac{\Delta x}{c \Delta t}$ and $\bar{\Delta} y=\frac{\Delta y}{c \Delta t}$, the non dimensional counterpart of the mesh size in $x$ and $y$ directions.

In the following we will detail the equations (25) in order to emphasize the use of the new boundary conditions (here we denoted by $w_{i, j}^{l}$ the $l$-th component of the vector $W_{i, j}$ ):

$$
\left\{\begin{aligned}
w_{i, j}^{1}-\frac{1-M_{n}}{\Delta x}\left(w_{i+1, j}^{1}-w_{i, j}^{1}\right) & +\frac{1}{\Delta y}\left[\frac{1+M_{t}}{2} w_{i, j}^{1}-\frac{1-M_{t}}{4} w_{i, j+1}^{1}-\frac{1+3 M_{t}}{4} w_{i, j-1}^{1}\right] \\
& +\frac{1}{\Delta y}\left[\frac{1-M_{t}}{2} w_{i, j}^{2}-\frac{1-M_{t}}{4} w_{i, j+1}^{2}-\frac{1-M_{t}}{4} w_{i, j-1}^{2}\right] \\
& +\frac{1}{\Delta y}\left[\frac{M_{t}}{\sqrt{2}} w_{i, j}^{3}+\frac{1-M_{t}}{2 \sqrt{2}} w_{i, j+1}^{2}-\frac{1+M_{t}}{2 \sqrt{2}} w_{i, j-1}^{3}\right]=f_{i, j}^{1} \\
w_{i, j}^{2}+\frac{1+M_{n}}{\Delta x}\left(w_{i, j}^{2}-w_{i-1, j}^{2}\right) & +\frac{1}{\Delta y}\left[\frac{1+M_{t}}{2} w_{i, j}^{2}-\frac{1-M_{t}}{4} w_{i, j+1}^{2}-\frac{1+3 M_{t}}{4} w_{i, j-1}^{2}\right] \\
& +\frac{1}{\Delta y}\left[\frac{1-M_{t}}{2} w_{i, j}^{1}-\frac{1-M_{t}}{4} w_{i, j+1}^{1}-\frac{1-M_{t}}{4} w_{i, j-1}^{1}\right] \\
& +\frac{1}{\Delta y}\left[\frac{M_{t}}{\sqrt{2}} w_{i, j}^{3}+\frac{1-M_{t}}{2 \sqrt{2}} w_{i, j+1}^{2}-\frac{1+M_{t}}{2 \sqrt{2}} w_{i, j-1}^{3}\right]=f_{i, j}^{2} \\
w_{i, j}^{3}+\frac{M_{n}}{\Delta x}\left(w_{i, j}^{3}-w_{i-1, j}^{3}\right) & +\frac{1}{\Delta y}\left[w_{i, j}^{3}-\frac{1-M_{t}}{2} w_{i, j+1}^{3}+\frac{1+M_{t}}{2} w_{i, j-1}^{3}\right] \\
& +\frac{1}{\Delta y}\left[\frac{M_{t}}{\sqrt{2}} w_{i, j}^{1}+\frac{1-M_{t}}{2 \sqrt{2}} w_{i, j+1}^{1}-\frac{1+M_{t}}{2 \sqrt{2}} w_{i, j-1}^{1}\right] \\
& +\frac{1}{\Delta y}\left[\frac{M_{t}}{\sqrt{2}} w_{i, j}^{2}+\frac{1-M_{t}}{2 \sqrt{2}} w_{i, j+1}^{2}-\frac{1+M_{t}}{2 \sqrt{2}} w_{i, j-1}^{2}\right]=f_{i, j}^{3} \\
& +\frac{M_{t}}{\Delta y}\left[w_{i, j}^{4}-w_{i, j-1}^{4}\right]=f_{i, j}^{4} . \\
w_{i, j}^{4}+\frac{M_{n}}{\Delta x}\left(w_{i, j}^{4}-w_{i-1, j}^{4}\right) &
\end{aligned}\right.
$$


As for the equations at $x=\gamma$ (i.e. for $i=l_{2}$ ), we use the last 3 equations of (26) but not the first

one because of the unknown $w_{l_{2}+1, j}^{1}$ which is not defined in the domain. We will provide the missing information from the boundary condition:

$$
a_{1} w_{l_{2}, j}^{1}+a_{2} w_{l_{2}, j}^{2}+a_{4} w_{l_{2}, j}^{4}=g_{j}, \forall j \in \mathbb{Z} .
$$

obtaining a linear system where the number of unknowns and the number of equations are the same.

For the discretized BVP in the domain $\left.\Omega_{2}=\right] \beta, \infty[\times \mathbb{R}$

$$
\left\{\begin{array}{l}
\frac{W}{c \Delta t}+A_{1} \frac{\partial W}{\partial x}+A_{2} \frac{\partial W}{\partial y}=f, \quad \text { for } x>\beta \\
\mathcal{A}^{\text {pos } W}=g \text { for } x=\beta
\end{array}\right.
$$

we obtain inside the domain the discrete equations (26). If we denote $\beta=l_{1} \Delta x$, on the points of the boundary, that is at $x=\beta$ (i.e. for $i=l_{1}$ ) we can only keep the first equation of (26) and add three more boundary conditions:

$$
\left\{\begin{array}{l}
-a_{2} w_{l_{1}, j}^{1}+\left(\lambda_{1}-\frac{a_{2}^{2}}{a_{1}}\right) w_{l_{1}, j}^{2}-\frac{a_{2} a_{4}}{a_{1}} w_{l_{1}, j}^{4}=g_{j, 2}, \forall j \in \mathbb{Z} . \\
\lambda_{3} w_{l_{1}, j}^{3}=g_{j, 3}, \forall j \in \mathbb{Z} . \\
-a_{4} w_{l_{1}, j}^{1}-\frac{a_{2} a_{4}}{a_{1}} w_{l_{1}, j}^{2}+\left(\lambda_{3}-\frac{a_{4}^{2}}{a_{1}}\right) w_{l_{1}, j}^{4}=g_{j, 4}, \forall j \in \mathbb{Z} .
\end{array}\right.
$$

\subsection{Optimization of the convergence rate for the discrete Schwarz algorithm}

Because of the linearity of the problem studied we can consider directly the algorithm applied to the homogeneous problem, in terms of the error vector. We will further look for a solution under the following form

$$
W_{i, j}=\sum_{k} \sum_{l=1}^{3} \alpha_{k l} e^{\left(i-\frac{1}{2}\right) \lambda_{l}(k) \Delta x} e^{I j k \Delta y} V_{l}(k)
$$

where $I^{2}=-1$. By introducing this expression into the discrete equation (25) we get that for each $k$, $\lambda_{l}(k)$ and $V_{l}(k)$ have to be the solution of

$$
\left(I d+\frac{\left|A_{1}\right|+A_{1}^{-} e^{\lambda_{l}(k) \Delta x}-A_{1}^{+} e^{-\lambda_{l}(k) \Delta x}}{\Delta x}+\frac{\left|A_{2}\right|+A_{2}^{-} e^{I k \Delta y}-A_{2}^{+} e^{-I k \Delta y}}{\Delta y}\right) V_{l}(k)=0 .
$$

If we denote by $L_{l}(k)=\frac{e^{-\lambda_{l}(k)}-1}{\Delta x}$ and by $e_{y}(k)=\frac{e^{I k \Delta y}-1}{\Delta x}$ and

$$
\left\{\begin{array}{l}
h_{1}(k)=-\frac{1-M_{t}}{4} e_{y}(k)+\frac{1+3 M_{t}}{4} \frac{e_{y}(k)}{e_{y}(k) \Delta y+1} \\
h_{2}(k)=-\frac{1-M_{t}}{4} e_{y}(k)+\frac{1-M_{t}}{4} \frac{e_{y}(k)}{e_{y}(k) \Delta y+1} \\
h_{3}(k)=\frac{1-M_{t}}{2 \sqrt{2}} e_{y}(k)+\frac{1+M_{t}}{2 \sqrt{2}} \frac{e_{y}(k)}{e_{y}(k) \Delta y+1} \\
h_{4}(k)=-\frac{1-M_{t}}{2} e_{y}(k)+\frac{1+M_{t}}{2} \frac{e_{y}(k)}{e_{y}(k) \Delta y+1}
\end{array}\right.
$$


and by doing the calculation we can see that the discrete eigenvalues $L_{l}(k)$ and the corresponding eigenvectors $V_{l}(k)=\left[V_{l, 1}(k), V_{l, 2}(k), V_{l, 3}(k), 0\right]^{t}, l=1,2,3$ satisfy the following:

$$
\left\{\begin{array}{l}
V_{l, 1}(k)\left[1-\left(1-M_{n}\right) L_{l}(k)\right]+h_{1}(k) V_{l, 1}+h_{2}(k) V_{l, 2}(k)+h_{3}(k) V_{l, 3}(k)=0 \\
V_{l, 2}(k)\left[1+\frac{1+M_{n}}{L_{l}(k) \Delta x+1}\right]+h_{1}(k) V_{l, 2}+h_{2}(k) V_{l, 1}(k)+h_{3}(k) V_{l, 3}(k)=0 \\
V_{l, 3}(k)\left[1+\frac{M_{n}}{L_{l}(k) \Delta x+1}\right]+h_{4}(k) V_{l, 3}+h_{3}(k) V_{l, 1}(k)+h_{3}(k) V_{l, 2}(k)=0 \\
V_{4}(k)=[0,0,0,1]^{t}
\end{array}\right.
$$

We note that $\lim _{\Delta x, \Delta y \rightarrow 0} L_{l}(k)=\lambda_{l}(k)$ where $\lambda_{l}(k)$ are the eigenvalues of the matrix $\mathcal{M}(k)$ given by (9) ands $V_{l}(k)$ can be found up to a multiplicative constant. Therefore $L_{l}(k)$ and $V_{l}(k)$ can be seen as the discrete counterpart of the eigenvalues and eigenvectors obtained in the continuous case. Moreover, $L_{l}(k)$ cannot be expressed analytically in a simple form as it is the root of a third order polynomial $P\left(L_{l}(k), \Delta x, \Delta y\right)$ whose expression is not detailed here but whose coefficients tend, as $\Delta x$ and $\Delta y$ tend to zero, to those of the characteristic polynomial of the matrix $\mathcal{M}(k)$. Thus, we can conclude using continuity arguments, that for a small $\Delta x$ and $\Delta y, P\left(L_{l}(k), \Delta x, \Delta y\right)$ possesses the same number of roots with positive real part as $\lim _{\Delta x, \Delta y \rightarrow 0} P\left(L_{l}(k), \Delta x, \Delta y\right)$.

The discrete counterpart of the Schwarz algorithm is:

$$
\begin{aligned}
& \Omega_{1} \text { : } \\
& \begin{array}{ll} 
\begin{cases}\frac{W_{i, j}^{p+1}}{c \Delta t} & +\frac{\left|A_{1}\right| W_{i, j}^{p+1}+A_{1}^{-} W_{i+1, j}^{p+1}-A_{1}^{+} W_{i-1, j}^{p+1}}{\Delta x} \\
& +\frac{\left|A_{2}\right| W_{i, j}^{p+1}+A_{2}^{-} W_{i, j+1}^{p+1}-A_{2}^{+} W_{i, j-1}^{p+1}}{\Delta y}=f, i<l_{2} . \\
\mathcal{A}^{\text {neg }} W_{i, j}^{p+1} & =\mathcal{A}^{\text {neg }} W_{i, j}^{p}, i=l_{2}\end{cases} \\
\Omega_{2}:
\end{array} \\
& \begin{cases}\frac{W_{i, j}^{p+1}}{c \Delta t} & +\frac{\left|A_{1}\right| W_{i, j}^{p+1}+A_{1}^{-} W_{i+1, j}^{p+1}-A_{1}^{+} W_{i-1, j}^{p+1}}{\Delta x} \\
& +\frac{\left|A_{2}\right| W_{i, j}^{p+1}+A_{2}^{-} W_{i, j+1}^{p+1}-A_{2}^{+} W_{i, j-1}^{p+1}}{\Delta y}=f, i>l_{1} . \\
\mathcal{A}^{p o s} W_{i, j}^{p+1} & =\mathcal{A}^{p o s} W_{i, j}^{p}, i=l_{1}\end{cases}
\end{aligned}
$$

where $\gamma=l_{2} \Delta x$ and $\beta=l_{1} \Delta x$.

If we assume that the flow is subsonic that is, if we adopt the same hypotheses as in section 2 then, in each subdomain, the solution has the form

$$
\begin{aligned}
W_{i, j} & =\sum_{k} \alpha_{k 1} e^{\left(i-\frac{1}{2}\right) \lambda_{1}(k) \Delta x} e^{I j k \Delta y} V_{1}(k) \text { for } i \leq l_{2} . \\
W_{i, j} & =\sum_{k}\left(\alpha_{k 2} e^{\left(i-\frac{1}{2}\right) \lambda_{2}(k) \Delta x} e^{I j k \Delta y} V_{2}(k)\right. \\
& \left.+\alpha_{k 3} e^{\left(i-\frac{1}{2}\right) \lambda_{3}(k) \Delta x} e^{I j k \Delta y} V_{3}(k)+\alpha_{k 4} e^{\left(i-\frac{1}{2}\right) \lambda_{3}(k) \Delta x} e^{I j k \Delta y} V_{4}(k)\right) \text { for } i \geq l_{1} .
\end{aligned}
$$

By introducing these expressions in the interface conditions of (29) we get the discrete convergence rate 
Table 1: Overlapping Schwarz algorithm

Numerical vs. theoretical parameters

\begin{tabular}{|c|c|c|c|c|}
\hline$M_{n}$ & $b_{1}^{\text {th }}$ & $b_{2}^{\text {th }}$ & $b_{1}^{\text {num }}$ & $b_{2}^{\text {num }}$ \\
\hline 0.1 & 1.6 & -0.8 & 1.6 & -0.9 \\
0.2 & 1.3 & -0.5 & 1.4 & -0.6 \\
0.3 & 1.25 & -0.3 & 1.25 & -0.45 \\
0.4 & 1.08 & -0.15 & 1.08 & -0.28 \\
0.5 & 1.03 & -0.08 & 1.02 & -0.23 \\
0.6 & 1.0 & 0.0 & 1.0 & 0.0 \\
0.7 & 1.02 & 0.06 & 1.01 & 0.04 \\
0.8 & 1.03 & 0.08 & 1.02 & 0.06 \\
0.9 & 1.06 & 0.08 & 1.04 & 0.06 \\
\hline
\end{tabular}

$(31)$

$$
\begin{aligned}
\rho_{2}^{2}\left(k, \Delta x, M_{n}, M_{t}\right) & =\mid \frac{\left(\left(v_{31}^{3}-v_{31}^{1}\right) b_{2}+\left(v_{31}^{1} v_{21}^{3}-v_{31}^{3} v_{21}^{1}\right) b_{1}\right)\left(b_{1}\left(M_{n}-1\right)+b_{2} v_{21}^{2}\left(M_{n}+1\right)\right)}{\left(\left(v_{31}^{3}-v_{31}^{2}\right) b_{2}+\left(v_{31}^{2} v_{21}^{3}-v_{31}^{3} v_{21}^{2}\right) b_{1}\right)\left(b_{1}\left(M_{n}-1\right)+b_{2} v_{21}^{1}\left(M_{n}+1\right)\right)} E_{2} \\
& -\frac{\left(\left(v_{31}^{2}-v_{31}^{1}\right) b_{2}+\left(v_{31}^{1} v_{21}^{2}-v_{31}^{2} v_{21}^{1}\right) b_{1}\right)\left(b_{1}\left(M_{n}-1\right)+b_{2} v_{21}^{3}\left(M_{n}+1\right)\right)}{\left(\left(v_{31}^{3}-v_{31}^{2}\right) b_{2}+\left(v_{31}^{2} v_{21}^{3}-v_{31}^{3} v_{21}^{2}\right) b_{1}\right)\left(b_{1}\left(M_{n}-1\right)+b_{2} v_{21}^{1}\left(M_{n}+1\right)\right)} E_{3} \\
& +\frac{\left(\left(b_{1}^{2}-b_{1}\right)\left(1-M_{n}\right)-b_{2}^{2}\left(M_{n}+1\right)\right)\left(v_{31}^{1}\left(v_{21}^{3}-v_{21}^{2}\right)+v_{31}^{3}\left(v_{21}^{2}-v_{21}^{1}\right)+v_{31}^{2}\left(v_{21}^{1}-v_{21}^{3}\right)\right)}{\left(\left(v_{31}^{3}-v_{31}^{2}\right) b_{2}+\left(v_{31}^{2} v_{21}^{3}-v_{31}^{3} v_{21}^{2}\right) b_{1}\right)\left(b_{1}\left(M_{n}-1\right)+b_{2} v_{21}^{1}\left(M_{n}+1\right)\right)} E_{3} \mid,
\end{aligned}
$$

where we denoted by $v_{j 1}^{i}=\frac{V_{i, j}(k)}{V_{i, 1}(k)}$ and $E_{j}=e^{-\left(\lambda_{j}(k)-\lambda_{1}(k)\right)\left(l_{2}-l_{1}\right) \Delta x}, j=2,3$.

Optimizing the convergence rate with respect to the two parameters is already a very difficult task at the continuous level in the non-overlapping case; we could not carry on such a process and obtaining analytical results at the discrete level in the overlapping case (which is our case of interest). Therefore, we will get the theoretical optimized parameters at the discrete level by means of a numerical algorithm, by calculating the following

$$
\begin{aligned}
& \rho\left(b_{1}, b_{2}\right)=\max _{k \in \mathcal{D}_{h}} \rho_{2}^{2}\left(k, \Delta x, M_{n}, M_{t}, b_{1}, b_{2}\right) \\
& \min _{\left(b_{1}, b_{2}\right) \in \mathcal{I}_{h}} \rho\left(b_{1}, b_{2}\right)
\end{aligned}
$$

where $\mathcal{D}_{h}$ is a uniform partition of the interval $[0, \pi / \Delta x]$ and $\mathcal{I}_{h} \subset \mathcal{I}$ a discretization by means of a uniform grid of a subset of the domain of the admissible values of the parameters. This kind of calculations are done once for all for a given pair $\left(M_{n}, M_{t}\right)$ before the beginning of the Schwarz iterations. An example of such a result is given in the Figure 1 for Mach number $M_{n}=0.2$. The computed parameters from the relation (32) will be further referred to with a superscript th. The theoretical estimates are compared afterwards with the numerical ones obtained by running the Schwarz algorithm with different pairs of parameters which lie in a an interval such that the algorithm is convergent. We are thus able to estimate the optimal values for $b_{1}$ and $b_{2}$ from these numerical computations. These values will be referred to by a superscript num.

\section{Implementation and numerical results}

We present here a set of results of numerical experiments that are concerned with the evaluation of the influence of the interface conditions on the convergence of the non-overlapping Schwarz algorithm of the 


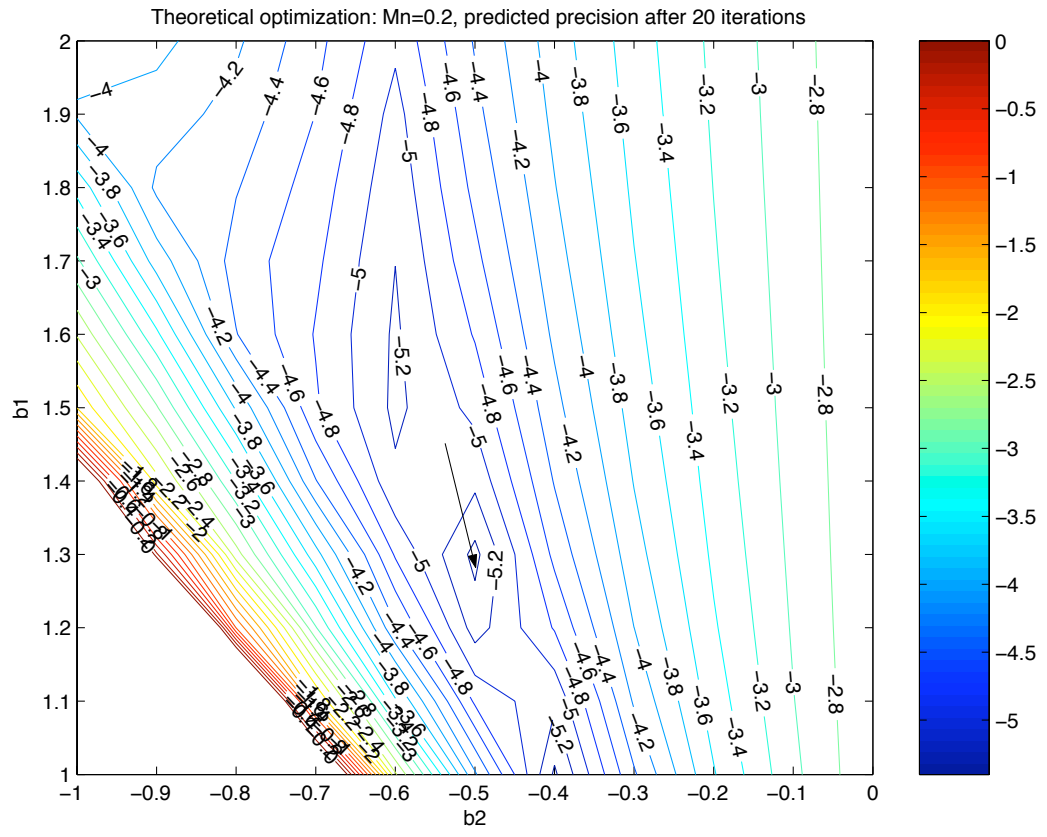

Figure 1: Isovalues of the predicted reduction factor of the error after 20 iterations via formula (32)

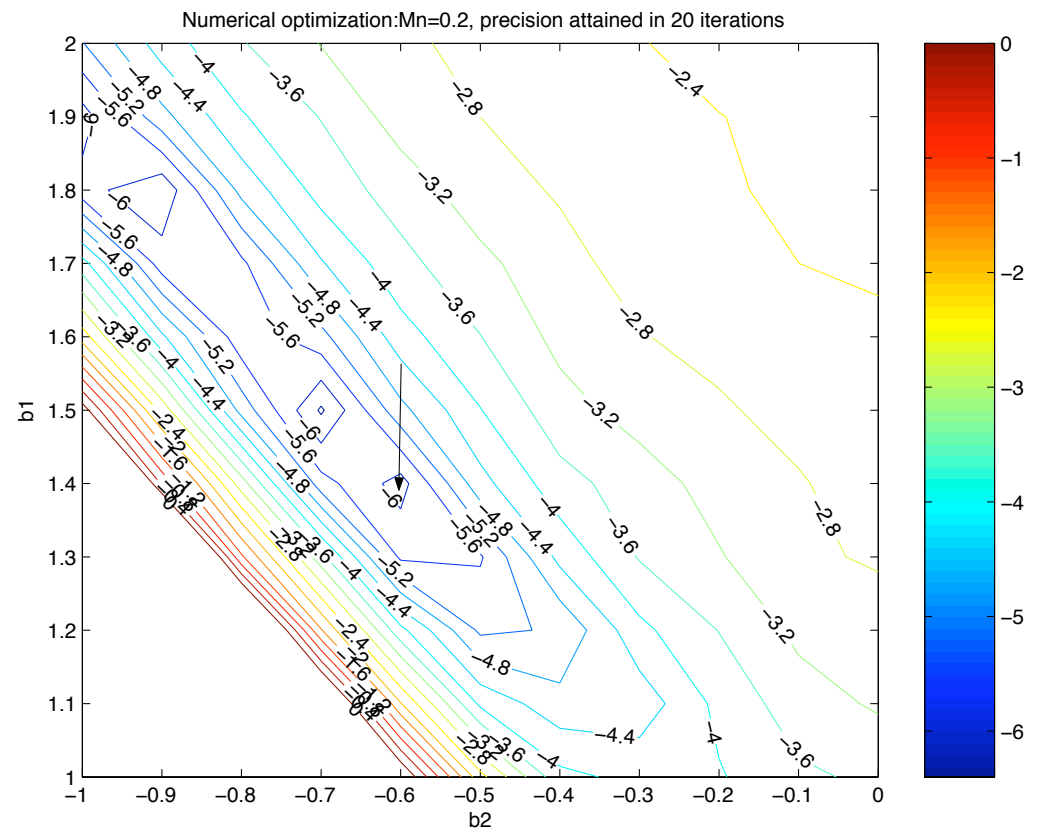

Figure 2: Isovalues of the reduction factor of the error after 20 iterations for the finite volume code 
Table 2: Overlapping Schwarz algorithm

Classical vs. optimized counts for different values of $M_{n}$

\begin{tabular}{|c|c|c|}
\hline$M_{n}$ & $I T_{0}^{\text {num }}$ & $I T_{o p}^{\text {num }}$ \\
\hline 0.1 & 48 & 19 \\
0.2 & 41 & 20 \\
0.3 & 32 & 20 \\
0.4 & 26 & 19 \\
0.5 & 22 & 18 \\
0.7 & 20 & 16 \\
0.8 & 22 & 15 \\
0.9 & 18 & 12 \\
\hline
\end{tabular}

form. The computational domain is given by the rectangle $[0,1] \times[0,1]$. The numerical investigation is limited to the resolution of the linear system resulting from the first implicit time step using a Courant number $\mathrm{CFL}=100$.

In all these calculations we considered a model problem: a flow normal to the interface (that is when $\left.M_{t}=0\right)$. In figures 1 and 2 we can see an example of a theoretical and numerical estimation of the reduction factor of the error (result of the optimization for different values of the normal Mach number is presented in Table 1). We illustrate here the level curves which represent the log of the precision after 20 iterations for different values of the parameters $\left(b_{1}, b_{2}\right)$, the minimum being attained in this case for $b_{1}^{\text {th }}=1.3$ and $b_{2}^{\text {th }}=-0.5, b_{1}^{\text {num }}=1.4$ and $b_{2}^{\text {num }}=-0.6$. We can see that we have good theoretical estimates of these parameters we can therefore use them in the interface conditions of the Schwarz algorithm.

Table 2 summarizes the number of Schwarz iterations required to reduce the initial linear residual by a factor $10^{-6}$ for different values of the reference Mach number with the optimal parameters $b_{1}^{\text {num }}$ and $b_{1}^{n u m}$. Here we denoted by $I T_{0}^{n u m}$ and $I T_{o p}^{n u m}$ the observed (numerical) iteration number for classical and optimized interface conditions in order to achieve a convergence with a threshlod $\varepsilon=10^{-6}$. The same results are presented in Figure 4. In the Figure 3 we compare the theoretical estimated iteration number in the classical and optimized case. Comparing figures 3 and 4 we can see that the theoretical prediction are very close to the numerical tests.

The conclusion of these numerical tests is, on one hand, that the theoretical prediction is very close to the numerical results: we can get by a numerical optimization (32) a very good estimate of optimal parameters $\left.\left(b_{1}, b_{2}\right)\right)$. On the other hand, the gain, in number of iterations, provided by the optimized interface conditions, is very promising for low Mach numbers, where the classical algorithm doesn't give optimal results. We can note that the optimized convergence rate is monotone with respect to the normal Mach number while the classical one isn't. For bigger Mach numbers, for instance, those who are close to 1 , the classical algorithm already has a very good behaviour so the optimization is less useful. In the same time we studied here the zero order and therefore very simple transmission conditions. The use of higher order conditions (see [GMN02]) is a possible way that can be further studied to obtain even better convergence results. 


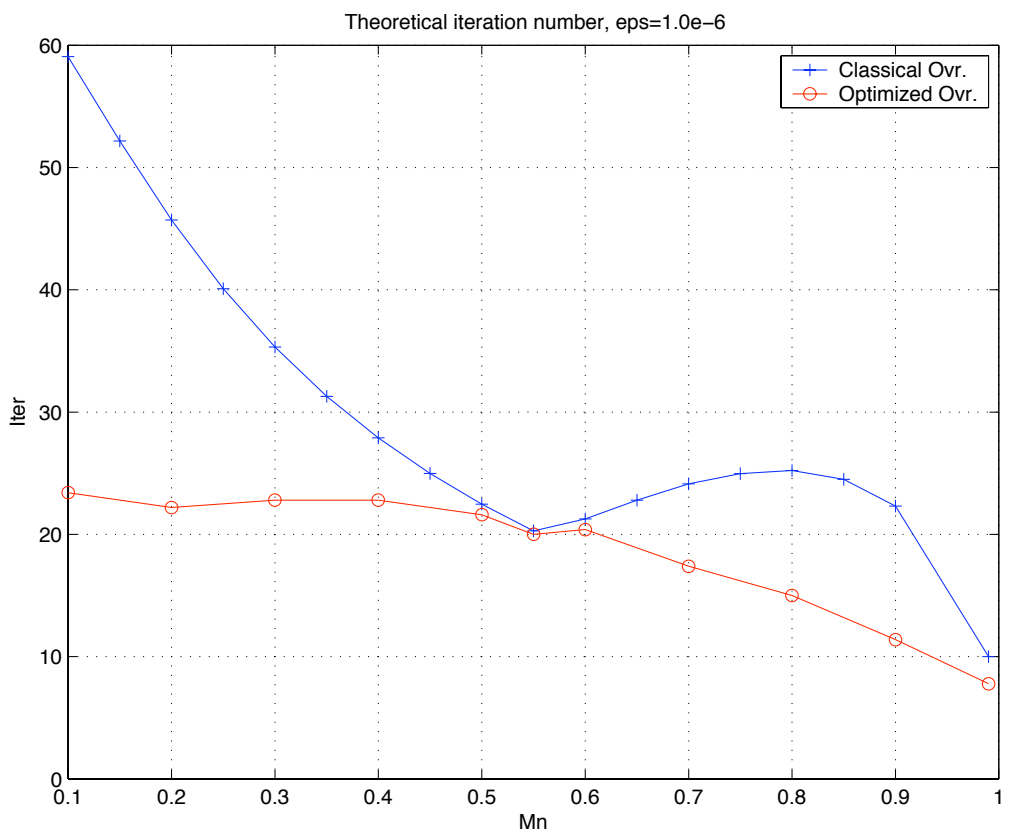

Figure 3: Theoretical iteration number: classical vs. optimized conditions

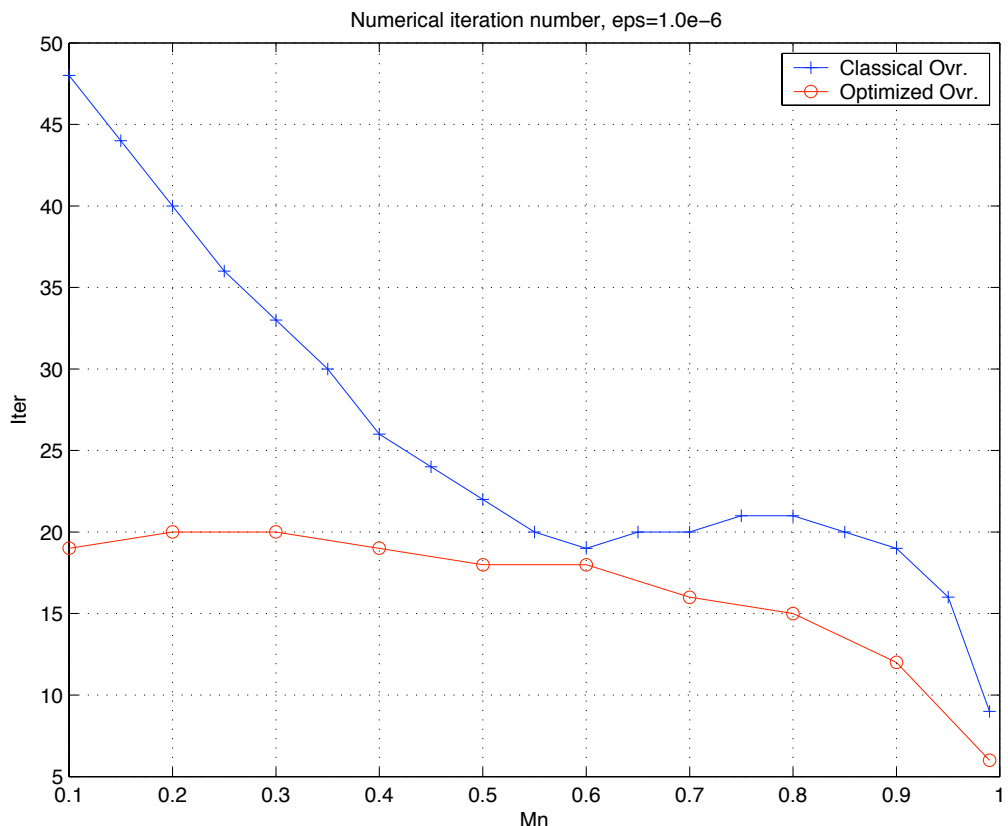

Figure 4: Numerical iteration number: classical vs. optimized conditions 


\section{Appendix}

In the following we place ourselves in the case $M_{t}=0$, therefore the convergence rate has the expression:

$$
\rho\left(R, b_{1}, b_{2}\right)=1-\frac{4 R b_{1}(1+M R)}{\left(R\left(b_{1}+b_{2}\right)+b_{1}-b_{2}\right)^{2}(1+M)}
$$

where $R=\sqrt{1+\xi^{2}\left(1-M^{2}\right)}$ is a real quantity depending only on the wave number $\xi$ and the Mach number $M=M_{n}$. We have the following optimization result:

Theorem 3 The optimization problem

$$
\min _{\left(b_{1}, b_{2}\right) \in \mathcal{I}_{1} \times \mathcal{I}_{2}} \max _{R \geq 1}\left|\rho\left(R, b_{1}, b_{2}\right)\right|
$$

possesses an analytical solution if $M \in\left[0.125, \frac{-5+3 \sqrt{17}}{16}\right]$ which is given by

$$
\left\{\begin{array}{l}
b_{1}=1+\frac{(\sqrt{M+1}-\sqrt{M})^{2}}{8 \sqrt{M(M+1)}} \\
b_{2}=\frac{\sqrt{M(M+1)}-(1-M)}{(\sqrt{M+1}+\sqrt{M}) \sqrt{M+1}} b_{1}
\end{array}\right.
$$

Before proceeding to the proof we will first formulate some properties of the convergence rate:

Lemma 2 We will denote by $R_{s}$ the local extremum (solution of the equation $\left.\rho_{R}^{\prime}\left(R, b_{1}, b_{2}\right)=0\right)$ when it exists. If the convergence rate has two distinct roots (there exists $R_{1,2}>1$ such that $\rho\left(R_{1}, b_{1}, b_{2}\right)=$ $\left.\rho\left(R_{2}, b_{1}, b_{2}\right)=0\right)$ then the solution of the optimization problem satisfies the relations:

$$
\max \left\{\rho\left(1, b_{1}, b_{2}\right), \rho\left(\infty, b_{1}, b_{2}\right)\right\}=-\rho\left(R_{s}, b_{1}, b_{2}\right)
$$

and this equation gives an admissible solution $\left(\left(b_{1}, b_{2}\right) \in D\right)$ only for $M \in\left[0.125, M_{0}\right] \equiv[0.125,0.55]$. Moreover the optimum verifies $\rho\left(1, b_{1}, b_{2}\right)=\rho\left(\infty, b_{1}, b_{2}\right)$ for $M \in\left[0.125, \frac{-5+3 \sqrt{17}}{16}\right]$.

Proof Before proceeding to the analysis we notice that for a fixed value $R>1$ the convergence rate grows monotonically in the parameters $b_{1,2}$ :

$$
\begin{aligned}
\frac{\partial \rho}{\partial b_{1}} & =\frac{4(M R+1) R\left(R b_{1}-R b_{2}+b_{1}+b_{2}\right)}{\left(\left(R b_{1}+R b_{2}+b_{1}-b_{2}\right)^{3}(1+M)\right)}>0 \\
\frac{\partial \rho}{\partial b_{2}} & =\frac{8(M R+1) R b_{1}(R-1)}{\left(\left(R b_{1}+R b_{2}+b_{1}-b_{2}\right)^{3}(1+M)\right.}>0
\end{aligned}
$$

When $\rho$ has two roots $R_{1,2}>1$ then the parameters satisfy the following

$$
b_{2} \in \mathcal{I}_{2}^{\prime}\left(b_{1}\right)=\left[\sqrt{\frac{4 M b_{1}}{M+1}}-b_{1}, \frac{M b_{1}-\sqrt{b_{1}\left(b_{1}-1\right)}}{M+1}\right]
$$

Moreover it admits one extremum $R_{s}$ which is the solution of its derivative:

$$
R_{s}=\frac{b_{1}-b_{2}}{b_{1}+b_{2}-2 M\left(b_{1}-b_{2}\right)}
$$


By imposing that $R_{s}$ be bigger than 1 we get an additional condition on $b_{2}$ :

$$
b_{2} \leq \frac{2 M+1}{M+1}-b_{1}
$$

which gives together with $b_{2} \in \mathcal{I}_{2}^{\prime}\left(b_{1}\right)$ a new restriction on $b_{1}$ :

$$
\left.\left.b_{1} \in \mathcal{I}_{1}=\right] 1, \frac{(2 M+1)^{2}}{4 M(M+1)}\right]
$$

The convergence rate for this value $\left(R_{s}\right)$ will have an opposite sign to

$$
\rho\left(1, b_{1}, b_{2}\right)=\frac{b_{1}-1}{b_{1}}>0
$$

and its modulus decreases monotonically in the parameters $b_{1,2}$ :

$$
\begin{aligned}
& \frac{\partial\left|\rho\left(R_{s}, b_{1}, b_{2}\right)\right|}{\partial b_{1}}=\frac{(M-1) b_{1}^{2}-(M+1) b_{2}^{2}}{\left(b_{1}-b_{2}\right)^{2}\left(M b_{1}-M b_{2}-b_{1}-b_{2}\right)^{2}(1+M)}<0 \\
& \frac{\partial\left|\rho\left(R_{s}, b_{1}, b_{2}\right)\right|}{\partial b_{2}}=-\frac{2 b_{1}\left(M b_{1}-(M+1) b_{2}\right.}{\left(b_{1}-b_{2}\right)^{2}\left(M b_{1}-M b_{2}-b_{1}-b_{2}\right)^{2}(1+M)}<0
\end{aligned}
$$

From the previous remarks we deduce that the positive quantities $\rho\left(1, b_{1}, b_{2}\right)$ and $\rho\left(\infty, b_{1}, b_{2}\right)$ are increasing with respect to the parameters $b_{1}$ and $b_{2}$ and $-\rho\left(R_{s}, b_{1}, b_{2}\right)$ is decreasing. Therefore $\rho$ is minimized if the "positive" maximum of $\rho$ and the "negative" maximum are equal in modulus, that is:

$$
\max \left\{\rho\left(1, b_{1}, b_{2}\right), \rho\left(\infty, b_{1}, b_{2}\right)\right\}=-\rho\left(R_{s}, b_{1}, b_{2}\right)
$$

The fact that $\rho\left(1, b_{1}, b_{2}\right)=\rho\left(\infty, b_{1}, b_{2}\right)$ comes out by supposing the contrary, that is for example:

$$
\rho\left(1, b_{1}, b_{2}\right)>\rho\left(\infty, b_{1}, b_{2}\right)>0
$$

The arbitrary small changes $\delta b_{1}$ and $\delta b_{2}$ of the parameters lead to arbitrary small change in $\rho\left(\infty, b_{1}, b_{2}\right)$ which is unimportant and this inequality will be preserved. The changes in $\rho\left(1, b_{1}, b_{2}\right)$ and $\rho\left(R_{s}, b_{1}, b_{2}\right)$ are given by:

$$
\begin{aligned}
\delta \rho\left(1, b_{1}, b_{2}\right) & =\delta b_{1} \frac{\delta \rho}{\delta b_{1}}\left(1, b_{1}, b_{2}\right)+\delta b_{2} \frac{\delta \rho}{\delta b_{2}}\left(1, b_{1}, b_{2}\right) \\
\delta \rho\left(R_{s}, b_{1}, b_{2}\right) & =\delta R_{s} \frac{\delta \rho}{\delta R}\left(R_{s}, b_{1}, b_{2}\right)+\delta b_{1} \frac{\delta \rho}{\delta b_{1}}\left(R_{s}, b_{1}, b_{2}\right)+\delta b_{2} \frac{\delta \rho}{\delta b_{2}}\left(R_{s}, b_{1}, b_{2}\right) \\
& =\delta b_{1} \frac{\delta \rho}{\delta b_{1}}\left(R_{s}, b_{1}, b_{2}\right)+\delta b_{2} \frac{\delta \rho}{\delta b_{2}}\left(R_{s}, b_{1}, b_{2}\right)
\end{aligned}
$$

and the extremum will be now located in $R_{s}+\delta R_{s}$. From the relations (35) and (41) we see that we can decrease $b_{1}$ and increase $b_{2}$ such that $\rho\left(R_{s}, b_{1}, b_{2}\right)$ increases while $\rho\left(1, b_{1}, b_{2}\right)$ decreases which contradicts the fact that we had an optimum. Therefore the optimum is attained for those values of the parameters where the equalities (34) hold. In the case when, for a given Mach number this cannot be satisfied (the solution to these equations gives admissible values to the parameters only for certain values of the Mach numbers as we will see later) we still have the weaker condition given by (40). 
In the following we will determine which are the values of Mach number for which one of the relations $(34)$ or $(40)$ hold. In order to have an admissible solution we have to check first that $b_{2} \in \mathcal{I}_{2}\left(b_{1}\right)$ where $b_{1}$ and $b_{2}$ are the solutions of (34):

$$
\left\{\begin{array}{l}
b_{1}=1+\frac{(\sqrt{M+1}-\sqrt{M})^{2}}{8 \sqrt{M(M+1)}} \\
b_{2}=\frac{\sqrt{M(M+1)}-(1-M)}{(\sqrt{M+1}+\sqrt{M}) \sqrt{M+1}} b_{1}
\end{array}\right.
$$

By solving the inequality which characterizes this inclusion we get that it is possible only for the values of the Mach number lying in the interval $\left[\frac{1}{8}, \frac{-5+3 \sqrt{17}}{16}\right]=[0.125,0.46]$. When the Mach number doesn't lie in this interval we still have the equation (40) verified. We can distinguish two possible cases: Case 1a. $\rho\left(1, b_{1}, b_{2}\right)=-\rho\left(R_{s}, b_{1}, b_{2}\right)>\lim _{R \rightarrow \infty} \rho\left(R, b_{1}, b_{2}\right)$.

In this case by solving the first equation we get the value of the parameter $b_{2}$ in function of $b_{1}$ :

$$
b_{2}\left(b_{1}, M\right)=\frac{\left(4 M b_{1}-2 M-2 \sqrt{4 b_{1}^{2}-6 b_{1}+2}\right) b_{1}}{2\left(2 b_{1}-1\right)(1+M)}
$$

and the inequation gives

$$
b_{1}>1+\frac{(\sqrt{M+1}-\sqrt{M})^{2}}{8 \sqrt{M(M+1)}}
$$

Afterwards we have to check that for a given Mach number $b_{2}\left(b_{1}, M\right) \in \mathcal{I}_{2}\left(b_{1}\right) \cap \mathcal{I}_{2}^{\prime}\left(b_{1}\right)$. We find numerically that this is true only when $M \in[0.125,0.55]$ but we cannot get an analytical solution.

Case 1b. $-\rho\left(R_{s}, b_{1}, b_{2}\right)=\lim _{R \rightarrow \infty} \rho\left(R, b_{1}, b_{2}\right)>\rho\left(1, b_{1}, b_{2}\right)$.

After some tedious calculations we get that the solution of the min-max problem is found in the previous case.

Now we will proceed to the proof of the theorem:

Proof If $M \in] 0.125,0.55$ [, according to the results given by the lemma, the solution is given by a pair $\left(b_{1}, b_{2}\right)$ where $b_{1}$ lies in an interval given by

$$
\left\{b_{1} \mid b_{2}\left(b_{1}, M\right) \in \mathcal{I}_{2}\left(b_{1}\right) \cap \mathcal{I}_{2}^{\prime}\left(b_{1}\right)\right\}
$$

Moreover the value of the convergence rate is increasing with respect to $b_{1}$ :

$$
\frac{\partial \rho\left(1, b_{1}, b_{2}\right)}{\partial b_{1}}=\frac{1}{b_{1}^{2}}>0
$$

so the value of $b_{1}$ which minimizes the convergence rate will be given by:

$$
\begin{gathered}
\min \left\{\inf \left\{b_{1} \mid b_{2}\left(b_{1}, M\right) \in \mathcal{I}_{2}\left(b_{1}\right) \cap \mathcal{I}_{2}^{\prime}\left(b_{1}\right)\right\}, \inf \left\{b_{1} \mid b_{2}\left(b_{1}, M\right) \in \mathcal{I}_{2}\left(b_{1}\right) \cap \mathcal{I}_{2}^{\prime}\left(b_{1}\right)\right\}\right\}= \\
=\inf \left\{b_{1} \mid b_{2}\left(b_{1}, M\right) \in \mathcal{I}_{2}\left(b_{1}\right) \cap \mathcal{I}_{2}^{\prime}\left(b_{1}\right)\right\}
\end{gathered}
$$

Moreover if $M \in\left[0.125, \frac{-5+3 \sqrt{17}}{16}[\right.$ the infimum of the relation (44) is given by the formula (42).

\section{References}

[AG04] Ana Alonso-Rodrìguez and Luca Gerardo-Giorda. New non-overlapping domain decomposition methods for the harmonic Maxwell system. Technical Report 529, CMAP - Ecole Polytechnique, 2004. 
[Bjø95] M. Bjørhus. Semi-discrete subdomain iteration for hyperbolic systems. Technical Report 4, NTNU, 1995.

[CDJP97] P. Collino, G. Delbue, P. Joly, and A. Piacentini. A new interface condition in the nonoverlapping domain decomposition for the Maxwell equations. Comput. Methods Appl. Mech. Engrg., 148:195-207, 1997.

[CFS98] X.-C. Cai, C. Farhat, and M. Sarkis. A minimum overlap restricted additive Schwarz preconditioner and application in 3D flow simulations. In C. Farhat J. Mandel and X.-C. Cai, editors, Proceedings of the 10th Domain Decomposition Methods in Sciences and Engineering, volume 218 of Contemporary Mathematics, pages 479-485. AMS, 1998.

[Cle98] S. Clerc. Non-overlapping Schwarz method for systems of first order equations. Cont. Math, 218:408-416, 1998.

[DJR92] Bruno Després, Patrick Joly, and Jean E. Roberts. A domain decomposition method for the harmonic Maxwell equations. In Iterative methods in linear algebra (Brussels, 1991), pages 475-484, Amsterdam, 1992. North-Holland.

[DLN02] V. Dolean, S. Lanteri, and F. Nataf. Construction of interface conditions for solving compressible Euler equations by non-overlapping domain decomposition methods. Int. J. Numer. Meth. Fluids, 40:1485-1492, 2002.

[DLN04] V. Dolean, S. Lanteri, and F. Nataf. Convergence analysis of a Schwarz type domain decomposition method for the solution of the Euler equations. Appl. Num. Math., 49:153-186, 2004.

[GMN02] M.-J. Gander, F. Magoulès, and F. Nataf. Optimized Schwarz methods without overlap for the Helmholtz equation. SIAM J. Sci. Comput., 24-1:38-60, 2002.

[JN00] C. Japhet and F. Nataf. The best interface conditions for domain decomposition methods: absorbing boundary conditions. In L. Tourrette, editor, Artificial Boundary Conditions, with Applications to Computational Fluid Dynamics Problems, pages 348-373. Nova Science, 2000.

[JNR01] C. Japhet, F. Nataf, and F. Rogier. The Optimized Order 2 method. application to convectiondiffusion problems. Future Generation Computer Systems, 18:17-30, 2001.

[QS96] A. Quarteroni and L. Stolcis. Homogeneous and heterogeneous domain decomposition methods for compressible flow at high Reynolds numbers. Technical Report 33, CRS4, 1996.

[Qua90] A. Quarteroni. Domain decomposition methods for systems of conservation laws : spectral collocation approximation. SIAM J. Sci. Stat. Comput., 11:1029-1052, 1990.

[Roe81] P.L. Roe. Approximate Riemann solvers, parameter vectors and difference schemes. J. Comput. Phys., 43:357-372, 1981. 